\title{
Die Folketingswahl in Dänemark vom 6. Juni 2019: Klarer Sieg des linken Lagers
}

Article

Accepted Version

Arndt, C. (2020) Die Folketingswahl in Dänemark vom 6. Juni 2019: Klarer Sieg des linken Lagers. Zeitschrift für

Parlamentsfragen, 50 (4). pp. 777-790. ISSN 0340-1758, doi: https://doi.org/10.5771/0340-1758-2019-4-777 Available at https://centaur.reading.ac.uk/87517/

It is advisable to refer to the publisher's version if you intend to cite from the work. See Guidance on citing.

To link to this article DOI: http://dx.doi.org/10.5771/0340-1758-2019-4-777

Publisher: Nomos

All outputs in CentAUR are protected by Intellectual Property Rights law, including copyright law. Copyright and IPR is retained by the creators or other copyright holders. Terms and conditions for use of this material are defined in the End User Agreement.

\section{www.reading.ac.uk/centaur}

\section{CentAUR}

Central Archive at the University of Reading

Reading's research outputs online 
Die Folketingswahl in Dänemark vom 6. Juni 2019: Klarer Sieg des linken Lagers

\author{
Christoph Arndt
}

Nach der Folketingswahl 2015 bildete in Dänemark zunächst die rechtsliberale Partei Venstre allein die Regierung, die nach einem Jahr um Konservative und Liberale Allianz erweitert wurde. Beide Konstellationen wurden von der Dänischen Volkspartei toleriert. Das bürgerliche Lager wirkte jedoch aufgrund des ständigen Streits über die Steuerpolitik in der Öffentlichkeit zerstritten und konnte somit weder von der guten Konjunktur im Wahljahr noch von den umgesetzten Versprechen bei der Innen- und Zuwanderungspolitik profitieren. Somit verlor es bei der Folketingswahl 2019 seine Mehrheit an den Block aus Sozialdemokraten, Sozialliberalen, Sozialistischer Volkspartei und der Einheitsliste, der die Wahl überraschend deutlich gewann. Die Sozialdemokraten hatten während der Legislaturperiode einen Kurswechsel zu einer dezidiert restriktiven Zuwanderungspolitik vollzogen und ihr Abschneiden wurde europaweit mit Spannung erwartet, da sie in dieser Hinsicht als mögliches Vorbild für andere Parteien galten, um traditionelle Stammwähler zurückzugewinnen. Neue Ministerpräsidentin wurde die Sozialdemokratin Mette Frederiksen, die eine Alleinregierung anführt. Diese wird von den anderen linken Parteien toleriert, die allerdings eine fundamental andere Zuwanderungspolitik als die Sozialdemokraten verfolgen. Somit hat die neue Regierungskonstellation eine Sollbruchstelle für die Wahlperiode 2019 bis 2023.

\title{
1. Die Ausgangslage
}

Die Wahl zum dänischen Folketing am 18. Juni 2015 hatte den vier bürgerlichen Parteien, der rechtsliberalen Venstre (V), der nationalkonservativen Dänischen Volkspartei (DF), der wirtschaftsliberalen Liberalen Allianz (LA) und den Konservativen (K) - dem „blauen Block“ - zusammen die entscheidenden 90 Mandate gebracht, so dass ein hauchdünner Regierungswechsel möglich wurde. Die Wahl markierte aber zugleich einen politischen Erdrutsch, da die Dänische Volkspartei mit über 21 Prozent der Stimmen zum ersten Mal die größte bürgerliche Partei - noch vor 
der rechtsliberalen Venstre - wurde. Gleichzeitig zog mit der radikal-ökologischen Alternative eine weitere Partei in das nun aus neun Fraktionen bestehende Folketing ein. ${ }^{1}$ Dänemark wurde nach gescheiterten Koalitionsverhandlungen der bürgerlichen Parteien im Sommer 2015 zunächst ein Jahr von einer Minderheitsregierung der Venstre mit Ministerpräsident Lars Løkke Rasmussen regiert. Diese hatte bereits nach acht Monaten zwei ihrer Minister, Verteidigungsminister Carl Holst und Landwirtschaftsministerin Eva Kjer Hansen, aufgrund von Skandalen und Misstrauensvoten verloren.

Bis dato arbeitete Venstre meist mit den Sozialdemokraten (S) und der DF zusammen, so dass Konservative und Liberale Allianz sowie Parteien links der Sozialdemokratie ohne großen Einfluss im ersten Jahr der Wahlperiode blieben. Den ersten Haushalt konnte Venstre noch relativ geräuschlos mit DF, Liberaler Allianz und Konservativen durchsetzen. Dieser wurde als bürgerlich charakterisiert, da er einige kleinere Steuersenkungen, eine Wiedereinführung der Deckelung der Sozialhilfe (Kontanthjælpsloft) und Mehrausgaben für die Polizeiarbeit und Altenpflege vorsah und somit einige Wahlversprechen der Parteien im blauen Block umsetzte. ${ }^{2}$

Insgesamt war die Wahlperiode 2015 bis 2019 überwiegend von den Themen Asyl und Zuwanderung sowie von Reformvorhaben bei Sozialstaat und Steuern geprägt. Bei letzteren gelang es dem regierenden blauen Block nicht, eine gemeinsame Linie zu finden. Diese Uneinigkeit wurde Ende August 2016 zum ersten Mal deutlich, als L $\phi k k e$ Rasmussen den 2025-Plan vorstellte. Dieses ambitiöse Reformpaket sah Steuersenkungen, eine Erhöhung des Renteneintrittsalters, eine Deckelung des Kindergeldes, die Umwandlung der Studienförderung in einen Teilkredit und eine Asylnotbremse vor. ${ }^{3}$ Allerdings konnte der Ministerpräsident nicht auf die Unterstützung aller Parteien im blauen Block setzen. Der Streit entzündete sich vor allem an der von der LA geforderten Abschaffung des Spitzensteuersatzes - eine ihrer zentralen Wahlkampfforderungen. Venstre wollte den Spitzensteuersatz teilweise

\footnotetext{
${ }^{1}$ Für eine Analyse der letzten Folketingswahl und Regierungsbildung vgl. Christoph Arndt, Die Wahlen zum dänischen Folketing vom 18. Juni 2015: knapper bürgerlicher Sieg bei erdrutschartigen Wählerwanderungen, in: ZParl, 47. Jg. (2016), H. 4, S. 771 - 782.

2 Vgl. Blå Finanslov, in: Jyllands-Posten online vom 21. November 2015, http://jyllandsposten.dk/debat/leder/ECE8233072/B1\%C3\%A5-finanslov/ (Abruf am 11. April 2016); Finansministeriet, Aftale mellem regeringen, Dansk Folkeparti, Liberal Alliance og Det Konservative Folkeparti: Finansloven for 2016, Kopenhagen 2015.

${ }^{3}$ Vgl. Overblik: Her er hovedpunkterne i 2025-planen, in: Jyllands-Posten online vom 30. August 2016, http://jyllands-posten.dk/politik/ECE8959604/overblik-her-er-hovedpunkterne-i-2025planen/; Overblik: Det siger partierne om Løkkes 2025-plan, in: Jyllands-Posten online vom 30. August 2016, http://jyllands-posten.dk/politik/ECE8961740/overblik-det-siger-partierne-om-loekkes-2025plan/ (Abruf jeweils am 10. Mai 2019).
} 
reduzieren, während die DF dagegen zunächst Steuersenkungen bei niedrigen Einkommen forderte, danach aber kategorisch ablehnte. ${ }^{4}$

Nachdem Venstres Alleinregierung, die nur über 34 der 179 Mandate verfügte, zunehmend an ihre parlamentarischen Grenzen kam und die beiden kleineren Parteien im blauen Block, Konservative und Liberale Allianz, immer mehr Druck auf Ministerpräsident Løkke Rasmussen ausübten, entschloss sich dieser im November 2016, die beiden in seine Regierung aufzunehmen. ${ }^{5}$

Die Disziplinierung der LA in einer Koalition war hierbei auch eines von Løkke Rasmussens strategischen Zielen, da diese ihre Wahlkampfversprechen zuvor mit Erpressungsversuchen realisieren wollte. Die Volkspartei war im Sommer 2016 auch prinzipiell zum Eintritt in eine bürgerliche Regierung bereit, entschied sich aber aus wahlstrategischen Motiven gegen Ministerposten im Kabinett Rasmussens und verblieb als „Støtteparti““, das heißt fester Tolerierungspartner einer Minderheitsregierung. ${ }^{6}$ Die Konservativen, die seit ihrem äußerst schwachen Resultat (3,4 Prozent) bei der Folketingswahl 2015 um ihr langfristiges Überleben kämpfen, versuchten sich ebenfalls mit Forderungen nach Steuersenkungen sowie Kriminalitätsbekämpfung zu profilieren. Bei der Regierungserweiterung wurde der Vorsitzende der Konservativen, S $\phi r e n$ Pape Poulsen, Justizminister, während der Vorsitzende der Liberalen Allianz, Anders Samuelsen, Außenminister wurde und die Partei auch sonst keine Schlüsselministerien zur Realisierung ihrer Wahlversprechen einnahm. Die Konservativen hatten sich somit zumindest ein Ministerium gesichert, um ihre innenpolitischen Forderungen umsetzen zu können. ${ }^{7}$

Die Erweiterung der Regierung im Spätherbst 2016 hatte jedoch nicht den politischen Handlungsspielraum für Løkke Rasmussen so erweitert, dass er ambitionierte Reformvorhaben aus dem 2015-Plan im Haushalt für 2017 realisieren konnte. Lediglich

\footnotetext{
${ }^{4}$ Vgl. Katrine Villarreal Villumsen, Analytiker: Selvom der manes til ro er bekymringen stor i blå blok, Danmarks Radio vom 20. April 2016, https://www.dr.dk/nyheder/politik/analytiker-selvom-der-manestil-ro-er-bekymringen-stor-i-blaa-blok (Abruf am 11. Mai 2019).

${ }^{5}$ Vgl. Jakob Stig Jørgensen / Søren Domino / Morten Munkholm, Løkke opgiver smal Venstre-regering: Vil have Liberal Alliance og de Konservative med, in: Berlingske online vom 19. November 2016, https://www.berlingske.dk/politik/loekke-opgiver-smal-venstre-regering-vil-have-liberal-alliance-og-dekonserv; Emma Gelbjerg Hansen, Løkke inviterer blå partier til regeringsforhandlinger, in: JyllandsPosten online vom 19. November 2016, http://jyllands-posten.dk/politik/ECE9164659/loekke-invitererblaa-partier-til-regeringsforhandlinger/ (Abruf jeweils am 19. Juni 2019).

${ }^{6}$ Vgl. Tobias Hansen B $\phi d k e r$, Politisk analytiker: Thulesen Dahl ser stadig med frygt på, hvordan det gik for SF, in: Jyllands-Posten online vom 18. Juni 2016, http://jyllandsposten.dk/politik/ECE8777151/politisk-analytiker-thulesen-dahl-ser-stadig-med-frygt-paa-hvordan-detgik-for-sf/ (Abruf am 19. Juni 2019).

${ }^{7}$ Vgl. Thomas Larsen, Søren Pape på vej mod konservativt skæbnevalg, in: Berlingske online vom 22. September 2018, https://www.berlingske.dk/analyse-og-perspektiv/soeren-pape-paa-vej-modkonservativt-skaebnevalg (Abruf am 20. Juni 2019).
} 
einige kleinere Steuersenkungen hatten Venstre und LA auf der steuerpolitischen Habenseite zu verbuchen, während es Einigkeit bei Verschärfungen des Einbürgerungsund Zuwanderungsrechts gab. So wurde die sogenannte Asylnotbremse vereinbart, die automatische Abweisungen von Asylbewerbern an der Grenze im Falle einer Krisensituation vorsieht. ${ }^{8}$ Der Streit in der Steuerpolitik, vor allem ausgelöst durch die zentralen Wahlkampfversprechen der LA und den Überlebenskampf der Konservativen, verblieb letztlich als Konfliktfeld für den Großteil der Wahlperiode und beschädigte die Geschlossenheit des blauen Blocks in der Öffentlichkeit. ${ }^{9}$

Im Jahr 2016 nahm der von den Sozialdemokraten nach der Wahlniederlage 2015 eingeleitete Strategiewechsel in der Migrationspolitik Konturen an. ${ }^{10}$ So schlossen sie sich oftmals den Gesetzesvorschlägen der bürgerlichen Parteien bei Asyl und Zuwanderung an. Der Kurswechsel wurde öffentlich vom Fraktionsvorsitzenden Henrik Sass Larsen mit der gescheiterten Integration von nicht-westlichen Zuwanderern und den Folgekosten für den Sozialstaat begründet: „Unsere Analyse ist, dass alleine die wirtschaftliche Folge der jetzigen Anzahl von Flüchtlingen und Einwanderern innerhalb weniger Jahre jeglichen wirtschaftlichen Spielraum aufzehren würde. Nichtwestliche Zuwanderer sind historisch schlecht in den Arbeitsmarkt integrierbar, und dies gilt auch den Syrern, welche in diesen Zeiten zu uns kommen. Desto mehr, desto schwerer, desto teurer. (...). Eine Masseneinwanderung - wie in Schweden gesehen - wird das Fundament unserer Wohlfahrtsgesellschaft ökonomisch und sozial unterminieren." ${ }^{11}$ Sass Larsen, eine der zentralen Personen beim Kurswechsel, argumentierte zudem, dass die Themen Asyl, Integration und Zuwanderung eine strukturelle bürgerliche Mehrheit seit 1998 hervorgebracht hätten und die oftmals durch den traditionellen Koalitionspartner, die sozialliberale Radikale Venstre (RV), forcierte liberale Zuwanderungspolitik Stammwähler der Sozialdemokraten in den blauen Block getrieben habe.

\footnotetext{
${ }^{8} \mathrm{Vgl}$. Steen A. Jørgensen, De blå partier er enige om næste års finanslov, in: Jyllands-Posten online vom 18. November 2016, http://jyllands-posten.dk/politik/ECE9162702/de-blaa-partier-er-enige-om-naesteaars-finanslov/ (Abruf am 19. Juni 2019).

${ }^{9}$ Vgl. exemplarisch BT, Kommentatorer: Strid om finanslov skader hele blå blok, in: BT online vom 12. Dezember 2017, https://www.bt.dk/politik/kommentatorer-strid-om-finanslov-skader-hele-blaa-blok; Johan Blem Larsen, Strid om skattelettelser har spøgt i blå blok siden valget, Danmarks Radio vom 21. April 2016, https://www.dr.dk/nyheder/politik/strid-om-skattelettelser-har-spoegt-i-blaa-blok-sidenvalget (Abruf jeweils am 19. Juni 2019).

${ }^{10}$ Vgl. Christoph Arndt, a.a.O. (Fn. 1), S. 782.

${ }^{11}$ Vgl. Henrik Sass Larsen, Vi vil gøre alt for at begrænse antallet af ikkevestlige flygtninge og indvandrere, in: Politiken vom 18. Dezember 2015; Erik Holstein, Kongemageren fra Vestegnen, in: Altinget nr. 6 (forår) 2017, S. 34 - 39.
} 
Der Kurswechsel der Sozialdemokraten leitete im Februar 2017 auch die Öffnung der Partei gegenüber einer Zusammenarbeit mit der DF nach der nächsten Wahl ein. Die DF hatte ebenfalls die Möglichkeit einer zukünftigen S-DF Kooperation ins Spiel gebracht, nachdem beide Parteien die Erhöhung des Renteneintrittsalters im 2025-Plan von Løkke Rasmussen verhindert hatten. ${ }^{12}$ Die Sozialdemokraten setzten sich somit zunehmend von ihrem traditionellen Koalitionspartner Radikale Venstre ab, während die DF in sozio-ökonomischen Politikfeldern sich den Sozialdemokraten annäherte, was die internen Gegensätze im blauen Block zunehmend verdeutlichte. ${ }^{13}$

Einen Monat später wurde dies bei der 50. Verschärfung des Zuwanderungsrechts durch Zuwanderungsministerin Inger Stфjberg, der Verabschiedung der Asylnotbremse und dem sogenannten Bandenpaket weiter illustriert. Das Bandenpaket des konservativen Justizministers Søren Pape Poulsen beinhaltet umfassende Maßnahmen zur Bekämpfung von Bandenkriminalität - insbesondere bei Rockern und Zuwanderern aus muslimisch geprägten Ländern. ${ }^{14}$ Es wird unter anderem durch Kürzungen des Kindergeldes für Ausländer finanziert und führt restriktive Bedingungen für entlassene Strafgefangene aus dem Bandenmilieu ein, was bis zum Aufenthaltsverbot in bestimmten Kommunen geht. ${ }^{15}$ Das Paket wurde von den drei Regierungsparteien, der DF und den Sozialdemokraten ausgearbeitet und verabschiedet, wobei auch die linke SF, die Sozialistische Volkspartei, überraschend für Inhalte des Paketes stimmte.

Angesichts der politischen Lage wurden die Parteitage der Liberalen Allianz und der Sozialdemokraten im Frühjahr 2017 mit Spannung erwartet, da eine Rebellion der Basis bei den Themen Steuersenkungen (LA) und Strategiewechsel bei der Zuwanderungspolitik (S) prognostiziert wurde. Die LA trat auf ihrem Parteitag jedoch

\footnotetext{
${ }^{12}$ Vgl. Dansk Folkeparti og Socialdemokratiet afviser ikke hinanden, in: Jyllands-Posten vom 13. Februar 2017, http://jyllands-posten.dk/politik/ECE9364426/dansk-folkeparti-og-socialdemokratiet-afviser-ikkehinanden/ (Abruf am 13. Februar 2017).

${ }^{13}$ Vgl. Jesper Haue Hansen, Socialdemokraterne og Radikale Venstre kalder hinanden usolidariske, verdensfjerne og rabiate, in: Jyllands-Posten online vom 23. April 2016, http://jyllandsposten.dk/politik/ECE8605133/socialdemokraterne-og-radikale-venstre-kalder-hinanden-usolidariskeverdensfjerne-og-rabiate/ (Abruf am 19. Juni 2019), Flemming Christiansen, Sammen i regering? Mette Frederiksen og Kristian Thulesen Dahl i ny pardans, in: Politiken online vom 13. Februar 2017, https://politiken.dk/indland/art5832519/Mette-Frederiksen-og-Kristian-Thulesen-Dahl-i-ny-pardans (Abruf am 13. Februar 2017).

${ }^{14}$ Für eine Übersicht über den Verhandlungsverlauf Steen A. Jørgensen, Bandepakke på plads: En jernnæve mod organiseret kriminalitet, in: Jyllands-Posten online vom 24. März 2017, http://jyllandsposten.dk/politik/ECE9457846/bandepakke-paa-plads-en-jernnaeve-mod-organiseret-kriminalitet/ (Abruf am 24. März 2017).

${ }^{15}$ Vgl. Justitsministeriet, Aftale om bandepakke, 24. März 2017, http://www.justitsministeriet.dk/nyt-ogpresse/pressemeddelelser/2017/aftale-om-bandepakke; Anders Leonhard, Børnepenge til udlændinge skal spæde til bandepakke, in: Jyllands-Posten online vom 24. März 2017, http://jyllandsposten.dk/politik/ECE9458376/boernepenge-til-udlaendinge-skal-spaede-til-bandepakke/ (Abruf jeweils am 24. März 2017).
} 
überraschend geschlossen auf, und interne Kritik an der Parteiführung verstummte, obwohl die Partei keines ihrer weitgehenden Wahlkampfversprechen in der Steuerpolitik bis dahin hatte durchsetzen können.

Bei den Sozialdemokraten kam es zu einer historischen Abrechnung mit der fehlgeschlagenen Integrationspolitik und der als zu naiv aufgefassten Linie der Partei seit dem Einwanderungsgesetz von 1983 (Udlændingeloven). Die neue Führung um Mette Fredriksen und Henrik Sass Larsen erklärte, dass man die offensichtlichen Integrationsprobleme bei nicht-westlichen Zuwanderern zulange ignoriert und tabuisiert habe. Damit kritisierten sie gegenteilige Aussagen des ehemaligen Vorsitzenden und Ministerpräsidenten Poul Nyrup Rasmussen. Das auf dem Parteitag verabschiedete neue Grundsatzprogramm enthielt zum ersten Mal Kernpunkte zum Thema Zuwanderung ${ }^{16}$ und wurde von Kommentatoren als Annäherung an die DF interpretiert. ${ }^{17}$

Zur Mitte der Wahlperiode wurden dann auch bei Venstre Debatten über Strategie und Personal geführt, da der neue Finanzminister Kristian Jensen - er hatte bei der Regierungserweiterung den Venstre-Veteranen Claus Hjort Frederiksen abgelöst - als möglicher Nachfolger Løkke Rasmussens gehandelt wurde. ${ }^{18}$ Dieser hatte durch den im Vorfeld der letzten Folketingswahl aufgekommenen Spesenskandal nach wie vor schlechte Sympathiewerte und war daher parteiintern in die Kritik geraten. ${ }^{19}$ Zugleich gab Venstre einige weiterreichende Reformambitionen im Bereich Sozialstaat auf, da sich aufgrund des Streits zwischen DF und LA sowie den gelegentlichen taktischen Allianzen zwischen S und DF keine Mehrheiten mehr für weite Teile des 2025-Plans und andere größere Sozialreformen fanden. Von Mitte 2017 bis Mitte 2018 konzentrierte sich die Regierungsarbeit daher häufig auf die Bereiche Zuwanderung und innere Sicherheit, da man hier durch die Unterstützung der Sozialdemokraten klare Mehrheiten hatte.

\footnotetext{
${ }^{16}$ Vgl. S skriver partihistorie: Udlændinge med i principprogram, in: Berlingske online vom 23. April 2017, https://www.b.dk/politiko/s-skriver-partihistorie-udlaendinge-med-i-principprogram (Abruf am 23. April 2017).

$17 \mathrm{Vgl}$. für eine Übersicht Jyllands-Posten, Kommentator: S rykker tættere på DF med nyt principprogram, in: Jyllands-Posten online vom 23. April 2017, http://jyllandsposten.dk/politik/ECE9524577/kommentator-s-rykker-taettere-paa-df-med-nyt-principprogram/ (Abruf am 23. April 2017).

${ }^{18} \mathrm{Vgl}$. Andreas Karker, Kristian Jensen tæt på at være urørlig: ,Kun én ting kan fælde ham‘, in: BT online vom 2. Dezember 2016, https://www.bt.dk/politik/kristian-jensen-taet-paa-at-vaere-uroerlig-kunen-ting-kan-faelde-ham (Abruf am 21. Juni 2019).

${ }^{19}$ Vgl. Christoph Arndt, a.a.O. (Fn. 1), S. 775. Für die Sympathiewerte Rasmussens vgl. Cecilie Gormsen, Ny måling: Løkke når lavpunkt i popularitet, in: Altinget online vom 29. März 2017, https://www.altinget.dk/artikel/ny-maaling-loekke-naar-lavpunkt-i-popularitet (Abruf am 19. Juni 2019).
} 
Im Herbst und Winter 2017 flammte der Streit in der Steuerpolitik im bürgerlichen Lager im Zuge der anstehenden Verhandlungen für den Haushalt 2018 jedoch erneut auf. Die LA versuchte ein letztes Mal, ihre versprochene Senkung des Spitzensteuersatzes durchzusetzen, indem sie ankündigte, dem Haushalt 2018 nur zuzustimmen, wenn gleichzeitig eine Steuerreform binnen Jahresfrist von der Regierung und der DF verabschiedet werde. Dies wurde als Erpressung wahrgenommen und scheiterte am Widerstand von $\mathrm{V}$ und $\mathrm{DF}$, die ihrerseits Steuersenkungen für niedrige Einkommen und bessere Altenpflege im Haushaltsgesetz 2018 realisierten und somit die LA in der Öffentlichkeit demütigten. ${ }^{20}$ Als Folge gab die LA Ende Dezember 2017 ihre Forderungen nach einer Abschaffung oder zumindest einer deutlichen Reduzierung des Spitzensteuersatzes auf und lag in Umfragen folglich deutlich unter ihren 7,5 Prozent der letzten Wahl. ${ }^{21}$

Zum Jahreswechsel setzte Ministerpräsident Løkke Rasmussen mit seiner Neujahransprache 2018 Gegenkultur und Parallelgesellschaften bei nicht-westlichen Zuwanderern auf die politische Tagesordnung. ${ }^{22}$ Seine Kritik daran wurde zum Gegenstand des Regierungshandelns für 2018. So legte die Koalition ein weitreichendes Maßnahmenpaket gegen Parallelgesellschaften im Frühjahr vor. Vor allem von Venstre initiiert, sah es unter anderem Zuzugskontrollen für Sozialleistungsempfänger, Strafzonen mit erhöhten Strafen für bestimmte Delikte und eine bessere Verteilung von Kindern und Schülern aus benachteiligten Wohngebieten in Bildungseinrichtungen vor. ${ }^{23}$ Zudem wurde ein Bedeckungsverbot in der Öffentlichkeit und damit de facto ein von der DF lange gefordertes Burkaverbot sowie eine stärkere Verknüpfung von vorherigen Einzahlungen und dem Bezug von Sozialleistungen eingeführt.

\footnotetext{
${ }^{20}$ Für eine Übersicht über den Haushalt 2018, Anne Funch, 10 hovedpunkter i finansloven for 2018, in: Berlingske online vom 8. Dezember 2017, https://www.b.dk/nationalt/10-hovedpunkter-i-finansloven-for2018 (Abruf am 8. Dezember 2017), Steen A. Jørgensen, Samuelsen erkender: Sejrsikre Facebookopdateringer kostede historiske skattelettelser, in: Jyllands-Posten online vom 12. Januar 2018, https://jyllands-posten.dk/politik/ECE10198674/samuelsen-erkender-sejrsikre-facebookopdateringerkostede-historiske-skattelettelser/ (Abruf am 12. Januar 2018).

${ }^{21} \mathrm{Vgl}$. LA dropper krav om topskattelettelser i denne regeringsperiode, in: Jyllands-Posten online vom 22. Dezember 2017, https://jyllands-posten.dk/politik/ECE10143217/la-dropper-krav-omtopskattelettelser-i-denne-regeringsperiode/ (Abruf am 22. Dezember 2017).

${ }^{22}$ Vgl. Regeringen, Statsministerens Nytårstale, Statsminister Lars Løkke Rasmussens nytårstale 2018, Kopenhagen, https://www.regeringen.dk/statsministerens-nytaarstale/lars-loekke-rasmussens-nytaarstale1-januar-2018/ (Abruf am 2. Januar 2018); Thomas Larsen, Løkke klar til opgør med ghetto-Danmark, in: Berlingske vom 1. Januar 2018.

${ }^{23}$ Vgl. Regeringen, Ét Danmark uden parallelsamfund - Ingen ghettoer i 2030, März 2018, Økonomi- og Indenrigsministeriet, Kopenhagen; Jesper Hvass / Mie Louise Raatz / Michael Hjøllund, Løkke vil lave særregler mod parallelsamfund, in: Jyllands-Posten online vom 13. September 2017, http://jyllandsposten.dk/politik/ECE9867453/loekke-vil-lave-saerregler-mod-parallelsamfund/ (Abruf am 13. September 2017).
} 
Die Maßnahmen gegen die Parallelgesellschaften und gescheiterte Integration bei nichtwestlichen Zuwanderern waren einer der wenigen Fälle, in denen die Regierung Rasmussen mitsamt DF geschlossen auftrat und es keinen Streit im Vorfeld gab. Die Sozialdemokraten stimmten vielen Maßnahmen $\mathrm{zu}$ und konnten somit ihren Kurswechsel zu restriktiverer Zuwanderungs- und Innenpolitik immer glaubwürdiger vertreten, so dass dem blauen Block dieses Wahlkampfthema zunehmend genommen wurde. ${ }^{24}$

Nachdem der Haushalt für 2019, der weitere Verschärfungen im Ausländerrecht vorsah, relativ spät im Herbst 2018 verabschiedet worden war, begannen zum Jahreswechsel 2019 die Spekulationen über die Neuwahl des Folketings, die von Løkke Rasmussen spätestens zum 18. Juni 2019 angesetzt werden musste. Dies lähmte den Politikbetrieb, so dass nur wenige wesentliche Gesetze und Vorhaben umgesetzt wurden und überwiegend Spekulationen über Regierungskonstellationen und Personalwechsel bei den Parteien auf der politischen Tagesordnung standen.

\section{Wahlkampf und Themen}

Nachdem lange gemutmaßt worden war, ob die Wahl zeitgleich mit der Wahl zum Europäischen Parlament am 26. Mai 2019 stattfinden würde, entschied sich Ministerpräsident Løkke Rasmussen für den 5. Juni und reizte somit nahezu die Maximaldauer einer Wahlperiode von vier Jahren aus. Allerdings gab es aufgrund der Umfragen auch keinerlei Anreiz, eine vorzeitige Wahl auszuschreiben, da alle Institute seit Monaten einen Vorsprung des „roten Blocks“ auswiesen. ${ }^{25}$ Damit fiel die Wahl zum Europaparlament in die heiße Phase des Wahlkampfes zum Folketing.

Die EP-Wahl wurde zum Erfolg für Venstre, die überraschend die größte Partei vor den Sozialdemokraten wurde. ${ }^{26}$ Die DF wurde unter anderem für die finanziellen Unregelmäßigkeiten ihrer EP-Fraktion abgestraft, verlor die Hälfte ihrer Wähler und wurde nurmehr viertstärkste Kraft. Die linke SF konnte von der Klimadebatte

\footnotetext{
${ }^{24} \mathrm{Vgl}$. für eine Analyse Jens Reiermann / Torben K. Andersen, Nu er det de røde der vinder på udlændingepolitikken, in: Mandag Morgen online vom 25. November 2018, https://www.mm.dk/artikel/nu-er-det-de-roede-der-vinder-paa-udlaendingepolitikken (Abruf am 19. Juni 2019).

${ }^{25}$ Für eine Übersicht über alle Umfragen und die Stärke der Regierungsalternativen im Zeitverlauf vgl. Berlingske Barometer, https://www.berlingske.dk/blokkenes-historik.

26 Für das Wahlresultat der Europawahl in Dänemark vgl. Danmarks Statistik, http://www.dst.dk/valg/Valg1684426/valgopg/valgopgHL.htm (Abruf am 19. Juni 2019).
} 
profitieren, schnitt überraschend zweistellig ab und wurde drittstärkste Partei. LA und die radikal-ökologische Alternative erreichten katastrophale Ergebnisse und blieben ohne Mandate im EP.

Neben der durch die Europawahl weiter angefachten Klimadebatte prägten auch die Themen Gesundheit, Zuwanderung und Soziales den Wahlkampf zum Folketing, der allerdings als relativ inhaltsarm charakterisiert wurde, da es kaum markante Wahlversprechen der Parteien gab. ${ }^{27}$ Im Gegensatz dazu wurde über die Regierungszusammensetzung nach der Wahl heftig spekuliert. Nachdem die Sozialdemokraten eine Koalition mit der sozialliberalen RV ausgeschlossen hatten, fragten sich politische Beobachter, wie denn eine linke Parlamentsmehrheit in eine sozialdemokratisch geführte Regierung münden sollte. ${ }^{28}$ Die Sozialdemokraten vertraten seit längerer Zeit restriktive Positionen in der Zuwanderungspolitik und versprachen eine weitgehende Beibehaltung der Politik der Vorgängerregierung, während mit Ausnahme einiger SF-Positionen alle anderen Parteien des roten Blocks sich für eine Rückkehr zu einer liberalen Asyl- und Zuwanderungspolitik aussprachen und dies auch zum Teil mit ultimativen Forderungen unterstrichen. Die Zerstrittenheit des roten Blocks in diesen Fragen war zunächst neben der guten Konjunkturlage eine der wenigen Trumpfkarten für Løkke Rasmussens Minderheitsregierung im Wahlkampf, da der blaue Block zumindest auf diesem Politikfeld relative Geschlossenheit vorweisen konnte.

Bereits Ende 2018 kamen jedoch auch Spekulationen über eine sozialdemokratische Minderheitsregierung auf, die von der DF tolerieret werden könne, um den Einfluss der anderen linken Parteien in der Zuwanderungspolitik $\mathrm{zu}$ minimieren. Bei den Vorsitzenden von Venstre und DF, Lars Løkke Rasmussen und Kristian Thulesen Dahl, gab es zudem Gedankenspiele über große Koalitionen aus S und V oder S, DF und V falls die Wahl eine unübersichtliche parlamentarische Lage hervorbringen sollte. ${ }^{29}$ Dies

\footnotetext{
${ }^{27}$ Vgl. Erik Holstein, Ny måling: Den grønne dagsorden tager en suveræn førsteplads, in: Altinget online vom 13. Mai 2019, https://www.altinget.dk/artikel/ny-maaling-den-groenne-dagsorden-tager-ensuveraen-foersteplads; Anders Baksgaard, Her er partiernes mærkesager til valget, in: Politiken online vom 7. Mai, 2019, https://www.altinget.dk/artikel/holstein-en-valgkamp-uden-meget-politisk-substans (Abruf jeweils am 19. Juni 2019).

${ }^{28}$ Vgl. Marchen Neel Gjertsen, Midt i klima, bogstavleg og spærregrænser blev det alligevel et udlændingevalg, in: Jyllands-Posten online vom 4. Juni 2019; Pia Kjarsgaard, En S-V-DF-regering er 'det eneste rigtige', Danmarks Radio online vom 18. Mai 2019, https://www.dr.dk/nyheder/politik/folketingsvalg/pia-kjaersgaard-en-s-v-df-regering-er-det-eneste-rigtige (Abruf am 20. Juni 2019).

${ }^{29}$ Vgl. Thomas Sфgaard Rohde, Løkke smider bombe midt i valgkampen: Åbner for regering med Venstre og Socialdemokratiet, in: Berlingske vom 16. Mai 2019; Thulesen Dahl om S-V-DF-regering:
} 
trug zur öffentlichen Verunsicherung über die zukünftige Regierungskonstellation in Dänemark bei, obwohl der rote Block in allen Umfragen einen komfortablen Vorsprung hatte. Die Strategie der DF, auch für eine Zusammenarbeit mit den Sozialdemokraten offen zu sein, führte ebenfalls zu wachsender Unklarheit, ob die Partei noch Teil des blauen Blocks sei, da sie sich in der Vergangenheit immer als Garant für stabile bürgerliche Mehrheiten und Politik präsentiert hatte. ${ }^{30}$ Mit dem Jahreswechsel 2018/2019 war dies auch mit deutlich schwindenden Umfragewerten verbunden.

Eine weitere Unbekannte im Wahlkampf tauchte im Frühjahr 2019 auf, als der Rechtsanwalt Rasmus Paludan begann, islamkritische Demonstrationen in Kopenhagener Vorstädten zu organisieren, wobei es zu Koranverbrennungen, Tumulten und größeren Polizeiaufgeboten kam. ${ }^{31}$ Paludan erhielt dabei erhebliche öffentliche Aufmerksamkeit und entschloss sich zur Gründung seiner eigenen Partei, Stram Kurs. Diese erreichte überraschend schnell und unter Ausnutzung einer Gesetzeslücke die geforderten 20.981 Unterschriften für die Wahlzulassung und konnte somit zur Folketingswahl antreten. Erste Umfragen sahen die von allen anderen Parteien isolierte Rechtspartei über der Zweiprozenthürde. Ebenfalls am Rande der Sperrklausel balancierte die Partei Nye Borgerlige (Neue Bürgerliche, NB), eine andere, von unzufriedenen Konservativen während der Wahlperiode gegründete Rechtspartei, die das marktliberale Profil der LA mit dem nationalkonservativen der DF zu kombinieren suchte. Die NB hatte schon während der vergangenen Monate in zahlreichen Umfragen über der Sperrklausel gelegen und hatte aufgrund des Dauerstreits zwischen DF und LA realistische Chancen, unzufriedene rechtskonservative und wirtschaftsliberale Wähler ausreichend zu mobilisieren. Somit gab es zum ersten Mal zwei Parteien, die rechts der DF angesiedelt waren, die bei knapper Verfehlung der Sperrklausel dem bürgerlichen Lager entscheidende Mandate kosten würden, bei Einzug aufgrund ihrer ultimativen Forderungen jedoch schwer in die parlamentarische Arbeit des blauen Blocks einzubinden wären. Die Anzahl der zugelassenen Parteien war mit 13 die höchste seit 1990.

\footnotetext{
Mulighederne er rigtig gode, in: Avisen.dk online vom 22. Mai 2019, https://www.avisen.dk/thulesendahl-s-df-v-regering-vil-kunne-finde-loesni_552426.aspx (Abruf am 20. Juni 2019).

${ }^{30}$ Vgl. Lars Trier Mogensen, DF-toppen balancerer på kanten af det hurlumhej, de selv flygtede fra for 24 år siden, in: Information online vom 16. März 2019, https://www.information.dk/indland/2019/03/dftoppen-balancerer-paa-kanten-hurlumhej-flygtede-24-aar-siden (Abruf am 20. Juni 2019).

${ }^{31}$ Exemplarisch hierzu Michael Thykier / Kaare Kronberg Jensen / William Kristensen, Tumult ved Christiansborg: Æggekast mod Paludan og demonstrant i kanalen, in: Jyllands-Posten online vom 22. März 2019, https://jyllands-posten.dk/indland/ECE11271472/tumult-ved-christiansborg-aeggekast-modpaludan-og-demonstrant-i-kanalen/ (Abruf am 20. Juni 2019).
} 


\section{Das Wahlergebnis und Wählerwanderungen}

Die Wahl vom 5. Juni, die am Grundgesetztag Dänemarks (Grundlovsdag) abgehalten wurde, brachte einen klaren Regierungswechsel. Der blaue Block verlor seine parlamentarische Mehrheit deutlich und erreichte nur noch 79 Mandate - für eine Mehrheit sind 90 Mandate erforderlich. Der von den Sozialdemokraten angeführte rote Block erreichte auch ohne die Partei Alternative und nordatlantische Mandate 91 Sitze, so dass eine Regierung erstmals nach 2007 wieder eine deutlichere Mehrheit von mehr als 90 Sitzen ohne Einberechnung der nordatlantischen Mandate hat. ${ }^{32}$

\section{Tabelle 1: Ergebnisse der dänischen Folketingswahl vom 5. Juni 2019}

\begin{tabular}{|c|c|c|c|c|c|}
\hline & \multicolumn{3}{|c|}{ Parteien- und Kandidatenstimmen } & \multicolumn{2}{|c|}{$\begin{array}{l}\text { Veränderungen zur Wahl } \\
2015\end{array}$} \\
\hline & absolut & Prozent & Sitze & $\begin{array}{l}\text { Prozentpun } \\
\text { kte }\end{array}$ & Sitze \\
\hline Wahlberechtigte & 4.219 .537 & 100 & $175^{\mathrm{a}}$ & & \\
\hline Wahlbeteiligung & 3.569 .521 & 84,6 & & $-1,3$ & \\
\hline ungültig & 37.801 & & & & \\
\hline gültig & 3.531 .720 & $100^{\mathrm{b}}$ & & & \\
\hline Sozialdemokraten (S) & 914.882 & 25,9 & 48 & $-0,4$ & +1 \\
\hline Dänische Volkspartei (DF) & 308.513 & 8,7 & 16 & $-12,4$ & -21 \\
\hline Venstre (V) & 826.161 & 23,4 & 43 & $+3,9$ & +9 \\
\hline Einheitsliste (EL) & 245.100 & 6,9 & 13 & $-0,9$ & -1 \\
\hline Liberale Allianz (LA) & 82.270 & 2,3 & 4 & $-5,2$ & -9 \\
\hline Die Alternative $(\AA)$ & 104.278 & 3,0 & 5 & $-1,8$ & -4 \\
\hline Radikale Venstre (RV) & 304.714 & 8,6 & 16 & $+4,0$ & +8 \\
\hline $\begin{array}{l}\text { Sozialistische Volkspartei } \\
\text { (SF) }\end{array}$ & 272.304 & 7,7 & 14 & $+3,5$ & +7 \\
\hline Konservative Volkspartei (K) & 233.865 & 6,6 & 12 & $+3,2$ & +6 \\
\hline Christdemokraten (KD) & 60,944 & 1,7 & 0 & $+0,9$ & \\
\hline Nye Borgerlige (NB) & 83,201 & 2,4 & 4 & $+2,4^{\mathrm{c}}$ & $+4^{\mathrm{c}}$ \\
\hline Stram Kurs (SK) & 63,114 & 1,8 & 0 & $+1,8^{c}$ & \\
\hline Klaus Riskær Pedersen & 29.600 & 0,8 & 0 & $+0,8^{c}$ & \\
\hline Einzelbewerber & 2.774 & 0,1 & 0 & $\pm 0,0$ & \\
\hline
\end{tabular}

32 Die Färöer und Grönland entsenden jeweils zwei Abgeordnete in das Folketing, sie werden als nordatlantische Mandate bezeichnet. 
${ }^{\mathrm{c}}$ Nicht angetreten bei Wahl 2015.

Quelle: Danmarks Statistik, Endgültiges Wahlergebnis.

Zudem gab es markante Wählerwanderungen mit einigen klaren Siegern und Verlierern.

Die Dänische Volkspartei war der große Verlierer der Wahl. Sie büßte 12,4 Prozentpunkte ein, so dass ihre Fraktion von 37 auf 16 Abgeordnete schrumpfte. Die erreichten 8,7 Prozent markieren ihr schlechtestes Resultat seit der Wahl 1998 und sind absolut der größte Mandatsverlust einer Partei seit der Erdrutschwahl von 1973.

Die DF verlor Nachwahlanalysen zufolge in drei Richtungen. Der größte Teil ihrer ehemaligen Wähler ging zur rechtsliberalen Venstre, die damit ihre 2015 an die DF abgegebenen Wähler 2015 wieder zurückgewann. Die Sozialdemokraten konnten etwa jeden zehnten DF-Wähler von 2015 zu sich herüberziehen. Sie wurden somit für ihren restriktiven Kurs in der Zuwanderungspolitik belohnt und konnten den letztlich wahlentscheidenden Blockwechsel einer signifikant großen Wählergruppe bewirken. Ein Drittel der DF-Wähler ging an die weiter rechtsstehenden Parteien Nye Borgerlige und Stram Kurs, deren Wählerbasis zu mehr als 50 Prozent aus ehemaligen DF-Wählern besteht. ${ }^{33}$ Der Kurs des Parteivorsitzenden, Thulesen Dahl, sich mehrere Regierungsund Tolerierungsoptionen offen $\mathrm{zu}$ halten, ist damit komplett fehlgeschlagen. Somit verlor die DF deutlich, obwohl die Partei einige langjährige Forderungen wie ein Burkaverbot, niedrigere Sozialhilfe für nicht-westliche Zuwanderer und den Paradigmenwechsel in der Asylpolitik in der abgelaufenen Wahlperiode realisieren konnte. Abgeordnete kritisierten die DF-Führung nach der Wahl für die schlechte Kommunikation der umgesetzten Wahlversprechen und Politikinhalte, was die Position Thulesen Dahls und des Fraktionsvorsitzenden Peter Skaarup künftig schwächen könnte.

Die rechtsliberale Venstre gewann überraschend vier Prozentpunkte hinzu und eroberte ihre Position als stärkste bürgerliche Partei von der DF zurück, obwohl Ministerpräsident Løkke Rasmussens Popularitätsdefizit, das durch den Spesenskandal 2015 entstanden war, nach wie vor eine Belastung war. ${ }^{34}$ Venstre gewann in erster Linie ehemalige Wähler der DF und LA (vgl. Tabelle 2). Løkke Rasmussens Position wurde durch den überraschenden Zugewinn intern gestärkt, und er kündigte an, den

33 Eigene Berechnungen basierend auf Epinion Wahlnachumfrage für Danmarks Radio, https://www.dr.dk/nyheder/politik/vaelgervandringer\#D2019 (Abruf am 2. November 2019).

${ }^{34} \mathrm{Vgl}$. Steen A. Jørgensen, Løkke halter stadig langt efter Mette Frederikse, in: Jyllands-Posten online vom 6. März 2019, https://jyllands-posten.dk/politik/ECE11229047/loekke-halter-stadig-langt-eftermette-frederiksen/ (Abruf am 20. Juni 2019). 
Parteivorsitz der Venstre nicht niederzulegen, obwohl dies im Falle eines Regierungsverlustes erwartet worden war.

Bei den beiden kleineren Regierungsparteien, den Konservativen und der Liberalen Allianz, gab es ebenfalls einen klaren Gewinner und einen klaren Verlierer. Die Konservativen, die seit den letzten beiden Wahlen um ihre langfristige Existenz kämpfen, konnten erstmals seit 2007 wieder Stimmen gewinnen und verbesserten sich um 3,2 Prozentpunkte, so dass sie jetzt zwölf statt sechs Abgeordnete im Folketing stellen. Im Gegensatz dazu verlor die LA zwei Drittel ihrer Stimmen und Mandate, selbst ihr Parteivorsitzender Anders Samuelsen konnte seinen Wahlkreis nicht halten und trat am Tag nach der Wahl zurück. Der LA wurden zuvor von den Umfragen Verluste vorhergesagt, allerdings waren die desaströsen 2,3 Prozent, die die wirtschaftsliberale Partei noch erreichte, von keinem Analysten erwartet worden. Die Liberale Allianz mobilisierte nur ein knappes Viertel der Wähler von 2015 und verlor vor allem an Venstre, dazu gingen signifikante Teile ihrer Wählerschaft $\mathrm{zu}$ den Konservativen, der Radikalen Venstre und Nye Borgerlige (vgl. Tabelle 2). Sie wurde daher für ihre nicht umgesetzten Wahlversprechen und die von ihr oft verursachten Querelen im bürgerlichen Lager deutlich abgestraft.

Im roten Block konnten die Sozialdemokraten trotz leichter Stimmenverluste ein Mandat gewinnen und stellen nach wie vor die stärkste Fraktion im Folketing. Die großen Gewinner im roten Block waren die Radikale Venstre und SF, die beide ihre Fraktion verdoppeln konnten und nun 16 respektive 14 Abgeordnete stellen. Beide Parteien hatten allerdings auch als Juniorpartner der Sozialdemokraten erhebliche Verluste bei der Wahl 2015 erlitten. Die Zugewinne für die Sozialliberalen und die Sozialistische Volkspartei sind nach Wahlnachbefragungen zu einem beträchtlichen Teil ehemalige Wähler der Sozialdemokraten. ${ }^{35}$

Die linksradikale Einheitsliste verlor einen Prozentpunkt und ein Mandat, nachdem ihre bis dahin populäre Spitzenkandidatin Johanne Schmidt-Nielsen aufgrund des Rotationsprinzips der Partei nicht erneut kandidieren durfte. Die radikal-ökologische Alternative, die seit der letzten Wahl erstmals im Folketing repräsentiert ist, konnte im Gegensatz zu den Sozialliberalen und der SF nicht von der Klimadebatte profitieren und verlor ebenfalls. Ihre Fraktion reduzierte sich auf nur noch fünf Abgeordnete, nachdem

35 Vgl. Analysen der Wählerwanderungen von Danmarks Radio und TV2, https://www.dr.dk/nyheder/politik/vaelgervandringer\#D2019 sowie http://nyheder.tv2.dk/politik/2019-0614-mens-df-og-la-bloedte-gik-andre-frem-gaa-paa-opdagelse-i-vaelgernes-vandring\#LF (Abruf am 2. November 2019). 
sie während der gesamten Wahlperiode mit Personalquerelen und Skandalen zu kämpfen hatte. Daher hatten bereits vor der Wahl etwa die Hälfte ihrer bisherigen Abgeordneten und einige Mitglieder des Parteivorstandes angekündigt, nicht wieder anzutreten, was ihr Imageproblem verdeutlichte. ${ }^{36}$ Durch die Verluste der offiziell als blockunabhängig angetretenen Alternative ist der rote Block nicht auf ihre fünf Mandate angewiesen.

Insgesamt gab es einen Stimmentausch im roten Block. Die Sozialdemokraten gewannen Arbeiterstimmen von der DF, verloren aber linke und urbane Wähler an die anderen linken Parteien, die den restriktiven Kurs in der Zuwanderungspolitik ablehnten. Dennoch gelang es den Sozialdemokraten, die für einen Regierungswechsel notwendige blocküberschreitende Wählerwanderung zu mobilisieren. Die Radikale Venstre konnte laut Analysen des Wahlforschers Kasper Mфller Hansen ebenfalls etwa 33.000 Stimmen aus dem blauen Block erobern (vor allem von der LA) - dies ist neben den deutlichen Gewinnen der Sozialdemokraten von der DF eine wesentliche Grundlage der klarsten linken Mehrheit seit der Wahl 1971. ${ }^{37}$

Bei den Kleinparteien gelang es lediglich der rechtskonservativen Nye Borgerlige, die Sperrklausel von zwei Prozent zu überwinden oder mindestens ein Wahlkreismandat in einem der zehn Wahlkreise zu erreichen. ${ }^{38}$ Sie rekrutierte ihre Wähler fast ausschließlich bei den ehemaligen Anhängern der Dänischen Volkspartei und der Liberalen Allianz (vgl. Tabelle 2). Die als rechtsradikal angesehene Stram Kurs scheiterte ebenso wie die Christdemokraten knapp mit 1,8 respektive 1,7 Prozent an der Sperrklausel. Die Christdemokraten verfehlten dabei ein Direktmandat um weniger als 200 Stimmen Abstand im Wahlkreis Vestjylland knapp. ${ }^{39}$ Deutlich unter der Sperrklausel blieb die Partei des umstrittenen Unternehmers und ehemaligen EPAbgeordneten Klaus Riskar Pedersen, der nach Privatinsolvenzen und Haftstrafen ein politisches Comeback angestrebt hatte.

\footnotetext{
${ }^{36}$ Für eine Zusammenstellung vgl. Julie Laurs Bundgaard, Politisk kommentator: Alternativet har alvorlige image-problemer, in: BT online vom 30. März 2019, https://www.bt.dk/politik/politiskkommentator-alternativet-har-alvorlige-image-problemer (Abruf am 20. Juni 2019).

${ }^{37}$ Vgl. Morten Øyen, Vælgervandringer: Radikale har hentet over 33.000 nye vælgere i blå blok, in: Altinget online vom 4. Juni 2019, https://www.altinget.dk/artikel/vaelgervandringer-radikale-har-hentetover-33000-nye-vaelgere-i-blaa-blok, (Abruf am 17. Juni 2019). Bei der Wahl 1971 erreichten die klassisch linken Parteien (S, RV und SF) 114 Mandate.

38 Erreicht eine Partei mindestens ein Wahlkreismandat in den zehn Mehrpersonenwahlkreisen (Storkredse), so stehen ihr ebenfalls Ausgleichsmandate zu, so dass sie eine Fraktion im Folketing stellen kann.

${ }^{39}$ Vgl. Anders Redder, Kristendemokraterne nåede at sælge en sejr, der aldrig kom i hus, in: Kristeligt Dagblad online vom 6. Juni 2019, https://www.kristeligt-dagblad.dk/politik/kristendemokraterne-naaedesaelge-en-sejr-der-aldrig-kom-i-hus-0 (Abruf am 17. Juni 2019).
} 


\begin{tabular}{|c|c|c|c|c|c|c|c|c|c|c|c|}
\hline \multicolumn{12}{|c|}{$\begin{array}{l}\text { Tabelle 2: Wäh } \\
\text { Zeilenprozent) }\end{array}$} \\
\hline & & \multicolumn{10}{|c|}{ Wahl 2019c } \\
\hline & & $S$ & $\mathrm{DF}$ & $\mathrm{V}$ & EL & LA & $\AA$ & RV & $\mathrm{SF}$ & $\mathrm{K}$ & $\mathrm{NB}^{\mathrm{b}}$ \\
\hline \multirow{10}{*}{ 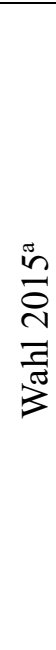 } & S & 79 & 1 & 3 & 2 & 0 & 1 & 4 & 7 & 1 & 0 \\
\hline & $\mathrm{DF}$ & 10 & 43 & 23 & 1 & 1 & 0 & 1 & 1 & 5 & 8 \\
\hline & V & 4 & 2 & 76 & 0 & 1 & 0 & 3 & 1 & 2 & 1 \\
\hline & EL & 8 & 1 & 0 & 68 & 0 & 4 & 3 & 15 & 1 & 0 \\
\hline & LA & 3 & 1 & 43 & 1 & 22 & 1 & 7 & 1 & 12 & 6 \\
\hline & $\AA$ & 12 & 0 & 2 & 17 & 0 & 28 & 18 & 19 & 1 & 0 \\
\hline & $\mathrm{RV}$ & 11 & 0 & 4 & 2 & 1 & 4 & 67 & 7 & 3 & 0 \\
\hline & $\mathrm{SF}$ & 17 & 0 & 2 & 5 & 0 & 2 & 5 & 64 & 0 & 0 \\
\hline & K & 4 & 0 & 12 & 0 & 0 & 2 & 4 & 0 & 74 & 0 \\
\hline & $\mathrm{NB}^{\mathrm{b}}$ & - & - & - & - & - & - & - & - & - & - \\
\hline \multicolumn{12}{|c|}{$\begin{array}{l}\text { a Prozentpunkte beziehen sich auf Wahlentscheidung in } 2019 \text { aufgeschlüsselt nach } \\
\text { Wahlentscheidung von } 2015 \text {. } \\
\text { b } 2015 \text { nicht angetreten. } \\
\text { ' Nur Parteien mit Mandaten im Folketing (Abkürzungen wie in Tabelle 1). } \\
\text { Quelle: Eigene Zusammenstellung basierend auf Epinion Wahlnachumfrage für Danmarks } \\
\text { Radio. }\end{array}$} \\
\hline
\end{tabular}

\section{Regierungs- und Oppositionsbildung}

Die Vorsitzende der Sozialdemokraten Mette Frederiksen wurde unmittelbar nach der Wahl in der Sondierungsrunde bei Königin Margrethe II mit der Bildung einer neuen Regierung beauftragt. Die Sozialdemokraten hatten bereits im Laufe der Wahlperiode angekündigt, eine Minderheitsregierung bilden zu wollen und somit erstmals seit 1983 eine Koalition mit der sozialliberalen Radikale Venstre ausgeschlossen. ${ }^{40}$ Strategisch strebte man eine Regierung an, die Wirtschafts- und Sozialpolitik primär mit den linken Parteien, Innen- und Zuwanderungspolitik mit den bürgerlichen Parteien führen sollte, um die neugewonnenen DF-Wähler zu halten sowie den Einfluss der RV bei Asyl- und Zuwanderung zu begrenzen, der für vorangegangene Niederlagen der Sozialdemokraten verantwortlich gemacht wurde.

\footnotetext{
${ }^{40}$ Vgl. Marchen Neel Gjertsen, Opbrud: Mette Frederiksen vil danne ren S-regering, in: Jyllands-Posten online vom 4. Juni 2018, https://jyllands-posten.dk/indland/ECE10653031/opbrud-mette-frederiksen-vildanne-ren-sregering/ (Abruf am 4. Juni 2018).
} 
Das Wahlergebnis ergab in dieser Hinsicht eine S-V-Mehrheit, die es den Sozialdemokraten zumindest theoretisch ermöglicht, die anderen linken Parteien mit einer alternativen Mehrheit unter Druck zu setzen. Die Regierungsverhandlungen erwiesen sich als schwierig und für dänische Verhältnisse langwierig, da die anderen Parteien des roten Blocks viele Forderungen aufstellten, um eine rein sozialdemokratische Minderheitsregierung zu tolerieren. Sie waren mit 20 Tagen die längsten Regierungs- und Tolerierungsverhandlungen seit $1981 .{ }^{41}$ Mette Frederiksen konnte dann in der Nacht zum 26. Juni 2019 eine Übereinkunft zwischen S, RV, SF und Einheitsliste zur Tolerierung einer sozialdemokratischen Minderheitsregierung und am nächsten Tag ihr neues Kabinett präsentieren. ${ }^{42}$ Abgesehen von dem ambitionierten Ziel, den $\mathrm{CO}_{2}$-Ausstoß in Dänemark bis 2030 um 70 Prozent zu reduzieren, verblieb die Übereinkunft jedoch häufig im Unklaren - beispielsweise bei der Finanzierung der von der SF geforderten versprochenen Minimumausstattung für Kindertagesstätten oder der von den Sozialdemokraten geplanten Frührente für körperlich besonders beanspruchte Berufsgruppen.

Bei der Zuwanderungspolitik unterstrichen die Sozialdemokraten ein Festhalten am Paradigmenwechsel der Vorgängerregierung, jedoch konnte die RV die Schließung eines umstrittenen Abschiebezentrums sowie ein Bleiberecht für Flüchtlinge mit langfristig gesichertem Arbeitsplatz durchsetzen. Im Bereich der Wirtschafts- und Sozialpolitik gab es lediglich Absichtserklärungen wie die von der Einheitsliste aufgestellte Forderung nach einem Verzicht auf „ungleichheitsschaffende Arbeitsmarktreformen“ oder ,zielgerichtete Steuererhöhungen“, was ihre Distanz zur RV widerspiegelt. Diese Distanz zwischen den Sozialliberalen und den Linkssozialisten in sozio-ökonomischen Fragen könnte neben der Zuwanderungspolitik damit eine Bruchstelle im roten Block werden - analog zu dem Dauerkonflikt von Volkspartei und Liberaler Allianz der vergangenen Wahlperiode.

Bei den Sozialdemokraten stellt sich zum einen die Frage, wie lange sie als Minderheitsregierung parlamentarisch überleben, wenn die übrigen Parteien im roten Block sich mit widerstreitenden Forderungen überbieten, ohne in eine Koalition

\footnotetext{
${ }^{41} \mathrm{Vgl}$. Kasper B. Andreasen, Regeringsdannelse tangerer historiske forhandlinger - få overblikket her, TV2 Nyheder vom 26. Juni 2019, http://nyheder.tv2.dk/politik/2019-06-25-regeringsdannelse-tangererhistoriske-forhandlinger-faa-overblikket-her (Abruf am 26. Juni 2019).

42 Vgl. Socialdemokratiet / Radikale Venstre / SF / Enhedslisten, Politisk forståelse mellem Socialdemokratiet, Radikale Venstre, SF og Enhedslisten: Retfærdig retning for Danmark, Christiansborg; Signe From Jensen, Her er aftalepunkterne i den politiske aftale mellem rød blok, in: Jyllands-Posten online vom 26. Juni 2019, https://jyllands-posten.dk/politik/ECE11466557/her-eraftalepunkterne-i-den-politiske-aftale-mellem-roed-blok/ (Abruf am 26. Juni 2019).
} 
eingebunden zu sein. Zum anderen lässt sich aus dem internationalen Vergleich fragen, ob der Ansatz der dänischen Sozialdemokratie, eine restriktive Migrationspolitik mit einer klassisch sozialdemokratischen Sozialpolitik zu kombinieren, zum Vorbild für andere sozialdemokratische Parteien wird, wie dies in Deutschland etwa vom ehemaligen SPD-Vorsitzenden Sigmar Gabriel angeregt wurde. ${ }^{43}$

Bei Venstre wird die kommende Wahlperiode zeigen, wie die Rechtsliberalen sich personell und strategisch für die Zukunft aufstellen. Nach der Wahl kam es bei Venstre rasch zu internen Querelen, die schliesslich zum Rücktritt Løkke Rasmussens vom Parteivorsitz und auch zum Rücktritt des langjährigen Kronprinzen Kristian Jensen als stellvertretendem Vorsitzenden auf einer außerordentlichen Vorstandssitzung im August 2019 führten. ${ }^{44}$ Auf dem anschliessenden ebenfalls außerordentlichen Parteitag am 21. September wurde der politische Sprecher und ehemalige Umweltminister, Jakob Ellemann-Jensen, zum neuen Venstre-Vorsitzenden gewählt. Die ehemalige, bei den Wählern populäre Integrationsministerin Inger Støjberg wurde in einer Kampfabstimmung zur stellvertretenden Vorsitzenden gewählt. Strategisch muss Venstre mit dem neuen Führungsduo die Regierungsfähigkeit des blauen Blocks wiederherstellen und die zerstrittenen und nun deutlich dezimierten Parteien DF und LA wieder in eine vom Wähler wahrgenommene kohärente bürgerliche Regierungsalternative einbinden. Bei der Liberalen Allianz dürfte hierbei jedoch zunächst eine klare wirtschaftsliberale Profilierung zu erwarten sein, um das langfristige Überleben der Partei zu sichern. Bei der Dänischen Volkspartei liegt der Fokus auf ein Wiederreichen der seit 2001 eigentlich stabilen Wählerunterstützung, indem sie sozialdemokratische Wähler anspricht, die von möglichen Zugeständnissen der Sozialdemokraten an die anderen linken Parteien abgeschreckt werden.

Im dänischen Parteiensystem könnte sich generell die Tendenz zur Fragmentierung und Volatilität weiter fortsetzen, wenn es nur noch Sozialdemokraten, Venstre und - bei einer möglichen Erholung - der DF gelingt, breitere Bevölkerungsschichten zu erreichen, während alle anderen Parteien aus der Opposition heraus zunehmend nur noch bei bestimmten Klientelen mit ultimativen Wahlversprechen ankommen und bei Regierungsbeteiligung abgestraft werden. Dies war bei SF, RV und LA als

\footnotetext{
${ }^{43}$ Vgl. Sigmar Gabriel, Wir brauchen den starken Staat zurück, in: Der Tagesspiegel vom 25. Juni 2019.

${ }^{44}$ Vgl. Ritzau, Overblik: Ballade i Venstre førte til Lars Løkkes exit, in: Jyllands-Posten online vom 16. November 2019, https://jyllands-posten.dk/politik/ECE11761270/overblik-ballade-i-venstrefoerte-til-lars-loekkes-exit/ (Abruf am 16. November 2019)
} 
Juniorpartner in den letzten beiden Wahlperioden der Fall, worauf sie jeweils Wähler an die radikaleren Neugründungen Alternative, Nye Borgerlige und Stram Kurs abgaben, so dass mittlerweile zehn Fraktionen im Folketing sitzen. 